\title{
Variational solution of Poisson's equation using plane waves in adaptive coordinates
}

\author{
José M. Pérez-Jordá* \\ Departament de Química Física, Universitat d'Alacant E-03080, Alacant, Spain \\ (Received 30 May 2014; published 13 November 2014)
}

\begin{abstract}
A procedure for solving Poisson's equation using plane waves in adaptive coordinates (u) is described. The method, based on Gygi's work, writes a trial potential $\xi$ as the product of a preselected Coulomb weight $\mu$ times a plane-wave expansion depending on $\mathbf{u}$. Then, the Coulomb potential generated by a given density $\rho$ is obtained by variationally optimizing $\xi$, so that the error in the Coulomb energy is second-order with respect to the error in $\xi$. The Coulomb weight $\mu$ is chosen to provide to each $\xi$ the typical long-range tail of a Coulomb potential, so that calculations on atoms and molecules are made possible without having to resort to the supercell approximation. As a proof of concept, the method is tested on the helium atom and the $\mathrm{H}_{2}$ and $\mathrm{H}_{3}{ }^{+}$molecules, where Hartree-Fock energies with better than milli-Hartree accuracy require only a moderate number of plane waves.
\end{abstract}

DOI: 10.1103/PhysRevE.90.053307

PACS number(s): 02.60.-x, 02.70.-c, 31.15.xt

\section{INTRODUCTION}

The use of plane waves in adaptive coordinates (PWACs) as basis functions for quantum-mechanical calculations was pioneered by Gygi [1,2]. This approach introduces a map $f$ from Cartesian coordinates $\mathbf{r}$ to some new adaptive coordinates $\mathbf{u}$,

$$
\mathbf{u}=f(\mathbf{r}) .
$$

Then this map is used to construct PWAC basis functions,

$$
\chi_{\mathbf{k}}=\left|\operatorname{det} \mathbf{J}_{f}\right|^{1 / 2} e^{i \mathbf{k} \cdot \mathbf{u}},
$$

where $\mathbf{k}$ is a wave vector and $\mathbf{J}_{f}$ is the Jacobian matrix of $f$. Finally, each molecular orbital is expressed as a linear combination of these PWAC bases,

$$
\psi=\sum_{\mathbf{k}} a_{\mathbf{k}} \chi_{\mathbf{k}}
$$

PWAC bases are orthogonal and complete [1,2].

Adaptive coordinates and PWAC bases have been used by a number of authors [1-19], and they promise some computational advantages over more conventional approaches, mainly Gaussian [20] or plane-wave [21] bases (although we would like to point out the existence of promising alternatives such as real-space mesh [22-27] or multiresolution wavelet $[28,29]$ techniques). Gaussians are accurate (allowing, for instance, routine all-electron calculations) but computationally demanding. Plane waves, on the other hand, are less accurate than Gaussians (so that pseudopotentials have to be used for core electrons), but they show, thanks to the fast Fourier transform [30] (FFT), a nicer scaling with the size of the system. Noting that a plane wave is just a PWAC basis function where the map $f$ is the identity map (that is, $\mathbf{u}=\mathbf{r}$ ), one would expect that a judicious choice of $f$ will make PWAC bases more accurate than plane waves, while hopefully maintaining their scaling advantages.

Gygi's original method $[1,2]$ has been applied mainly to periodic systems (for nonperiodic systems, calculations have been performed [3,4] by means of the supercell approximation

*jmpj@ua.es
[21]), and the problem posed by the nucleus-electron singularities has to be circumvented in an approximate way by the use of pseudopotentials [1-8] or some other approximation for the nuclear potential $[9-11,19]$. These limitations are being addressed by our ongoing project [14-17], the goal of which is to use PWAC bases in order to perform accurate all-electron calculation on nonperiodic systems. This paper is devoted to the development of a variational procedure for solving Poisson's equation and computing the Coulomb repulsion energy, which is a crucial ingredient of any quantum chemical calculation.

First we will review the work done so far, and then we will describe our proposed procedure for computing Coulomb energies via the solution of Poisson's equation. After that, we will discuss the technical implementation of the algorithm, and, as a proof of concept, we will compute the Hartree-Fock energy of the helium atom and the $\mathrm{H}_{2}$ and $\mathrm{H}_{3}{ }^{+}$molecules. We will close the paper with a summary of the main conclusions.

\section{REVIEW OF PREVIOUS WORK}

In this section, we will summarize our improvements [14-17] over Gygi's original method [1,2], beginning with the choice of the map $\mathbf{u}=f(\mathbf{r})$, which is of fundamental importance in calculations employing PWAC basis. The kind of maps $[14,17]$ that we are using have three main features:

(i) The domain for the Cartesian coordinates $\mathbf{r}$ covers the whole space, while the range of the adaptive coordinates $\mathbf{u}$ is kept within finite intervals. This enables the exact and trivial handling of isolated atoms or molecules, which are surrounded by an infinite amount of empty space, without having to resort to the supercell approach (we should point out that there are [31] efficient alternatives to the supercell approach, but based on wavelets rather than on plane waves).

(ii) The maps are adaptive, meaning that they adapt themselves to the molecular geometry, so that regions of chemical interest (such as atomic cores or bonds) are dealt with more carefully. This allows, for instance, the accurate description of core densities for heavy atoms such as uranium [14], thus rendering the use of pseudopotentials unnecessary, and all-electron calculations feasible. 
(iii) The maps can be inverted easily, so that the value $\mathbf{r}$ corresponding to a given $\mathbf{u}$ can be found efficiently. This is a crucial property, since the evaluation of molecular integrals requires extensive sampling in $\mathbf{u}$ space.

Apropos this sampling, it is well known that some quantum chemical methods (such as the Hartree-Fock model or density-functional theory) are variational, meaning that if $\delta \xi$ represents the error in the wave function, then the error in the total energy is much lower, only second-order in $\delta \xi$. The use of PWAC bases for such methods would, in principle, be variational too, provided that the computational implementation is accurate enough. A problem, however, is posed by the evaluation of molecular integrals, which have to be done by numerical integration over a given set of sampling points, so that, if this set is not large enough, a numerical error will be introduced that will kill the second-order advantage. We have solved $[15,17]$ this problem by implementing an efficient and accurate procedure that minimizes the numerical error, thus keeping the whole procedure truly variational and with a total energy error that is second-order in $\delta \xi$. As an illustration, we have presented [15] evidence that second-order variational calculations yield errors about one order of magnitude smaller that the corresponding first-order nonvariational counterparts. It should be noted that, by the nature of the maps used in some calculations [1-6] for periodic systems, this sampling procedure is not really necessary to obtain second-order errors for them.

This evaluation of molecular integrals by numerical integration is threatened by the nucleus-electron singularity that appears in nuclear potential integrals: if one of the sampling points is located at (or very near) one nucleus, then we will have a division by zero (or by a very small number) problem, which will ruin the accuracy of the whole calculation. We have solved [17] this problem by transforming the nuclear integrals via Green's first identity, so that the corresponding integrands are singularity-free. This procedure is exact, and there is no need to use pseudopotentials [1-8] or some other approximation for the nuclear potential [9-11,19].

These improvements are encouraging, and they have been successfully tested [17] on one-electron atoms and molecules. A necessary step for the extension of the method to arbitrary many-electron atoms and molecules is the evaluation of the electron-electron Coulomb repulsion energy. This will be accomplished in the present paper by improving an early prototype [15] solver for Poisson's equation, which was developed and tested on crude model densities (spherically averaged Hartree-Fock densities, conveniently screened to take away the long-range tail of the Coulomb potential). Improvements to this prototype will be aimed at getting the correct long-range behavior of the Coulomb potential and at selecting a map $\mathbf{u}=f(\mathbf{r})$ able to deal successfully both with Poisson's equation and Schrödinger's equation.

\section{COULOMB ENERGY VIA POISSON'S EQUATION}

The evaluation of the Coulomb energy is a crucial component of any quantum chemistry calculation. For example, in density-functional theory, given an electron density $\rho$ built from a set of molecular orbitals $\left\{\psi_{i}\right\}$,

$$
\rho=\sum_{i}\left|\psi_{i}\right|^{2}
$$

the Coulomb energy has the following expression:

$$
E_{\mathrm{ee}}=\frac{1}{2} \iint \frac{\rho(\mathbf{r}) \rho\left(\mathbf{r}^{\prime}\right)}{\left|\mathbf{r}-\mathbf{r}^{\prime}\right|} d \mathbf{r} d \mathbf{r}^{\prime}
$$

(we use atomic units). For simplicity, in this paper we will restrict ourselves to Coulomb energies having this form. Other quantum chemistry models, such as the Hartree-Fock method, have electron-electron repulsion energies that can also be expressed in terms of orbitals, so that the procedure explained here can be easily adapted to these other methods.

To get the Coulomb energy, one can first obtain the Coulomb potential $v$ generated by a density $\rho$,

$$
v(\mathbf{r})=\int \frac{\rho\left(\mathbf{r}^{\prime}\right)}{\left|\mathbf{r}-\mathbf{r}^{\prime}\right|} d \mathbf{r}^{\prime}
$$

and then compute the Coulomb energy as

$$
E_{\mathrm{ee}}=\frac{1}{2} \int \rho(\mathbf{r}) v(\mathbf{r}) d \mathbf{r} .
$$

Alternatively, instead of using Eq. (6), one can obtain the Coulomb potential by solving Poisson's equation,

$$
\nabla^{2} v=-4 \pi \rho \text {. }
$$

This is the approach that we will use here.

Let us have the following functional:

$$
F_{\rho}[\xi]=\frac{1}{8 \pi} \int|\nabla \xi|^{2} d \mathbf{r}-\int \rho \xi d \mathbf{r},
$$

where $\xi$ is a trial potential. It is easy to prove, via the Euler-Lagrange equation (see, for instance, Ref. [11]), that $v$, the solution of Poisson's equation, minimizes this functional: given a trial potential

$$
\xi=v+\delta \xi
$$

then

$$
F_{\rho}[\xi]=F_{\rho}[v]+\frac{1}{8 \pi} \int|\nabla \delta \xi|^{2} d \mathbf{r} \geqslant F_{\rho}[v]=-E_{\text {ee }} .
$$

Thus we can solve Poisson's equation via the minimization of $F_{\rho}$, and the value of $-F_{\rho}$ at the minimum will provide the value of the Coulomb energy. This procedure is variational, so that the error in the Coulomb energy is second-order in $\delta \xi$.

We propose the following expression for the trial potential:

$$
\xi=\mu(\mathbf{r}) C(\mathbf{u}),
$$

where the Cartesian coordinates $\mathbf{r}$ and the adaptive coordinates $\mathbf{u}$ are related by the map $\mathbf{u}=f(\mathbf{r}), C$ is an expansion in plane waves in $\mathbf{u}$ coordinates,

$$
C(\mathbf{u})=\sum_{\mathbf{k}} c_{\mathbf{k}} e^{i \mathbf{k} \cdot \mathbf{u}},
$$

and $\mu$ is the Coulomb weight, that is, a preselected weight function that would provide the appropriate boundary conditions (it will be discussed in depth below).

The electron density $\rho$ is built from molecular orbitals, as shown in Eq. (4). The molecular orbitals are expressed in 
turn as a linear combination of PWAC basis functions, as in Eq. (3). Therefore, the density will have, according to Eq. (2), an expression of the form

$$
\rho=\left|\operatorname{det} \mathbf{J}_{f}\right| \Omega(\mathbf{u}),
$$

where $\Omega$ is an expansion in plane waves in $\mathbf{u}$ coordinates,

$$
\Omega(\mathbf{u})=\sum_{\mathbf{k}} d_{\mathbf{k}} e^{i \mathbf{k} \cdot \mathbf{u}}
$$

(the single summation is justified by the fact that the product of two plane waves is another plane wave). It should be noted that, for the procedure to be practical, the same map $\mathbf{u}=$ $f(\mathbf{r})$ should be used for both the Coulomb potential and the molecular orbitals.

We now insert the expressions for $\xi$ and $\rho$ given, respectively, by Eqs. (12) and (14), into Eq. (9), and, using the map $\mathbf{u}=f(\mathbf{r})$ and the chain rule for the gradient operator $\nabla$, we rewrite, in a similar way as was done [17] for the Schrödinger equation, the functional $F_{\rho}[\xi]$ as a functional of $C, F_{\Omega, f}[C] \equiv F_{\rho}[\xi]$,

$$
\begin{aligned}
F_{\Omega, f}[C]= & \int\left[\sum_{j=1}^{3} \sum_{k=1}^{3} Q_{j k} C_{j} C_{k}+\sum_{j=1}^{3} Q_{j}\left(C_{j} C+C C_{j}\right)\right. \\
& \left.+Q C^{2}-\mu \Omega C\right] d \mathbf{u}
\end{aligned}
$$

with

$$
\begin{gathered}
C_{j}(\mathbf{u})=\frac{\partial C}{\partial u_{j}}, \\
Q(\mathbf{u})=\frac{\sum_{i=1}^{3}\left(\frac{\partial \mu}{\partial x_{i}}\right)^{2}}{8 \pi\left|\operatorname{det} \mathbf{J}_{f}\right|}, \\
Q_{j}(\mathbf{u})=\frac{\mu \sum_{i=1}^{3} \frac{\partial \mu}{\partial x_{i}} \frac{\partial u_{j}}{\partial x_{i}}}{8 \pi\left|\operatorname{det} \mathbf{J}_{f}\right|}, \\
Q_{j k}(\mathbf{u})=\frac{\mu^{2} \sum_{i=1}^{3} \frac{\partial u_{j}}{\partial x_{i}} \frac{\partial u_{k}}{\partial x_{i}}}{8 \pi\left|\operatorname{det} \mathbf{J}_{f}\right|},
\end{gathered}
$$

where $x_{1}, x_{2}$, and $x_{3}$ represent the Cartesian coordinates and $u_{1}, u_{2}$, and $u_{3}$ represent the adaptive coordinates,

$$
\begin{gathered}
\left(x_{1}, x_{2}, x_{3}\right) \equiv \mathbf{r}, \\
\left(u_{1}, u_{2}, u_{3}\right) \equiv \mathbf{u} .
\end{gathered}
$$

Finally, to complete the description of our algorithm, we have to provide an expression for the Coulomb weight $\mu$ introduced in Eq. (12). It is well known that the Coulomb potential $v$ generated by a molecular electron density has a long-range tail with the following form:

$$
\lim _{|\mathbf{r}| \rightarrow \infty} v \propto \frac{1}{|\mathbf{r}|}
$$

Therefore, a Coulomb weight $\mu$ with exactly this kind of behavior would be a sensible choice. We propose to make $\mu$ equal to the Coulomb potential generated by a single normalized Gaussian distribution $g_{\lambda_{C}}$ centered at the origin,

$$
\mu(\mathbf{r}) \equiv \mu_{\lambda_{C}}(r)=\int \frac{g_{\lambda_{C}}(s)}{|\mathbf{s}-\mathbf{r}|} d \mathbf{s}
$$

where

$$
g_{\lambda_{C}}(r)=\frac{1}{\lambda_{C}^{3} \sqrt{\pi^{3}}} e^{-\left(r / \lambda_{C}\right)^{2}},
$$

and where the parameter $\lambda_{C}$ determines the extent of the Gaussian. To compute $\mu_{\lambda_{C}}$, we use the relation [32]

$$
\mu_{\lambda_{C}}(r)=\frac{2}{\sqrt{\pi} \lambda_{C}} F_{0}\left(r^{2} / \lambda_{C}^{2}\right)
$$

where the function $F_{m}$ with $m$ integer (not to be confused with the functional $F_{\rho}$ ) is related [33] to the incomplete gamma function $\gamma$,

$$
F_{m}(t)=\frac{1}{2 t^{m+1 / 2}} \gamma(m+1 / 2, t) .
$$

For the derivatives of $\mu_{\lambda_{C}}$, it is easy to arrive at the following relation:

$$
\frac{\partial \mu_{\lambda_{C}}}{\partial x_{i}}=-\frac{4 x_{i}}{\sqrt{\pi} \lambda_{C}^{3}} F_{1}\left(r^{2} / \lambda_{C}^{2}\right),
$$

where $x_{i}$ represents any of the Cartesian coordinates.

\section{IMPLEMENTATION AND BENCHMARKING}

To minimize the functional $F_{\Omega, f}[C]$ in Eq. (16), we have to implement a procedure for finding out the coefficient $c_{\mathbf{k}}$ of each plane wave $e^{i \mathbf{k} \cdot \mathbf{u}}$ in Eq. (13). However, as Coulomb potentials and densities are real functions, we prefer to use cas functions,

$$
\operatorname{cas} x=\cos x+\sin x
$$

instead of complex exponential functions,

$$
e^{i x}=\cos x+i \sin x .
$$

Here "cas" stands for "cosine and sine." The corresponding $3 \mathrm{D}$ basis function will be

$$
\chi_{\mathbf{k}}=\frac{1}{(\sqrt{2 \pi})^{3}} \operatorname{cas}\left(k_{u_{1}} u_{1}\right) \operatorname{cas}\left(k_{u_{2}} u_{2}\right) \operatorname{cas}\left(k_{u_{3}} u_{3}\right),
$$

where the integer components of the wave vector $\mathbf{k} \equiv$ $\left(k_{u_{1}}, k_{u_{2}}, k_{u_{3}}\right)$ are restricted as follows:

$$
\begin{aligned}
& -\frac{N_{u_{1}}}{2} \leqslant k_{u_{1}}<\frac{N_{u_{1}}}{2}, \quad-\frac{N_{u_{2}}}{2} \leqslant k_{u_{2}}<\frac{N_{u_{2}}}{2}, \\
& -\frac{N_{u_{3}}}{2} \leqslant k_{u_{3}}<\frac{N_{u_{3}}}{2},
\end{aligned}
$$

and $N_{u_{1}}, N_{u_{2}}$, and $N_{u_{3}}$ give the size of the basis along the respective dimension, so that the total number of basis functions will be $N_{u_{1}} \times N_{u_{2}} \times N_{u_{3}}$. For simplicity, a basis of the same size will be used for the molecular orbitals, expressed as indicated in Eq. (3).

We proceed by writing the functional $F_{\Omega, f}[C]$ in matrix form, and we define a square matrix $\mathbf{A}$ and a column matrix $\mathbf{b}$ 
with respective elements,

$$
\begin{aligned}
(\mathbf{A})_{\mathbf{I} \mathbf{m}}= & 2 \int\left[\sum_{j=1}^{3} \sum_{k=1}^{3} Q_{j k} \frac{\partial \chi_{\mathbf{l}}}{\partial u_{j}} \frac{\partial \chi_{\mathbf{m}}}{\partial u_{k}}\right. \\
& \left.+\sum_{j=1}^{3} Q_{j}\left(\frac{\partial \chi_{\mathbf{l}}}{\partial u_{j}} \chi_{\mathbf{m}}+\chi_{\mathbf{l}} \frac{\partial \chi_{\mathbf{m}}}{\partial u_{j}}\right)+Q \chi_{\mathbf{l}} \chi_{\mathbf{m}}\right] d \mathbf{u}
\end{aligned}
$$

and

$$
(\mathbf{b})_{\mathbf{l}}=\int \mu \Omega \chi_{\mathbf{l}} d \mathbf{u} .
$$

These integrals are evaluated by the sampling procedure outlined in Sec. II, so that the numerical integration error is kept low enough to ensure an overall second-order variational procedure $[15,17]$. We have used $10 N_{u_{1}}, 10 N_{u_{2}}$, and $10 N_{u_{3}}$ sampling points along the respective dimension for $\mathbf{A}$, and $3 N_{u_{1}}, 3 N_{u_{2}}$, and $3 N_{u_{3}}$ for b. Next, taking into account Eqs. (16) and (13), we arrive at the following equivalence:

$$
F_{\Omega, f}[C]=\frac{1}{2} \sum_{\mathbf{l}} \sum_{\mathbf{m}} c_{\mathbf{l}}(\mathbf{A})_{\mathbf{l} \mathbf{m}} c_{\mathbf{m}}-\sum_{\mathbf{l}}(\mathbf{b})_{\mathbf{l}} c_{\mathbf{l}} .
$$

To minimize $F_{\Omega, f}[C]$, we equate its partial derivatives with respect to each $c_{1}$ to zero, and we obtain the following system of linear equations:

$$
\sum_{\mathbf{m}}(\mathbf{A})_{\operatorname{lm}} c_{\mathbf{m}}=(\mathbf{b})_{\mathbf{l}}
$$

As $\mathbf{A}$ is a symmetric positive-definite matrix, it is possible to solve this system by Cholesky decomposition and obtain the coefficients $c_{\mathbf{m}}$ (note that, although Cholesky decomposition is fine for systems of moderate size, more efficient procedures should be used for larger systems, such as conjugate-gradient or iterative methods).

The maps $\mathbf{u}=f(\mathbf{r})$ that will be used in this work are composites of two maps $g$ and $h$, that is, $f=g \circ h$, or

$$
\mathbf{u}=f(\mathbf{r})=h(g(\mathbf{r}))
$$

These composite maps have been successfully used [17] for solving the $3 \mathrm{D}$ Schrödinger equation. We will give a brief outline.

(i) The $g$ map depends [17] on three parameters: $\lambda_{x}, \lambda_{y}$, and $\lambda_{z}$ (one for each Cartesian coordinate), parameters that are related to the extent of the system, and therefore may have different values for different atoms and molecules.

(ii) The $h$ map is built [17] from a point-density $\rho_{h}$,

$$
\rho_{h}(\mathbf{s})=\sum_{i} p\left(\mathbf{s}-\mathbf{s}_{i}\right) .
$$

The peak function $p$ depends on three parameters: $L_{x}, L_{y}$, and $L_{z}$. To add some extra flexibility to our maps, we have introduced a new parameter $\gamma$ to this definition, and now we will use

$$
\rho_{h}(\mathbf{s})=\frac{1-\gamma}{8 \pi^{3}}+\gamma \sum_{i} p\left(\mathbf{s}-\mathbf{s}_{i}\right)
$$

instead of the original [17] form given in Eq. (38) (which corresponds to $\gamma=1$ ). For $\gamma=0$, the point-density $\rho_{h}$ becomes constant, which reduces $h$ to the identity map.

Summing up, the maps used in this work will depend on seven parameters: $\lambda_{x}, \lambda_{y}, \lambda_{z}, L_{x}, L_{y}, L_{z}$, and $\gamma$. It is important to stress that, in a calculation on a given molecule, the same map should be used to obtain the Coulomb potential (via Poisson's equation, as explained above), and to obtain the molecular orbitals (via the 3D Schrödinger equation [17]).

As a proof of concept, we will perform some restricted Hartree-Fock (RHF) calculations on two-electron systems. For two-electron systems, the electronic RHF energy [32] may be obtained by minimizing the following functional:

$$
\begin{aligned}
E_{\mathrm{RHF}}[\tilde{\psi}]= & 2 \int \tilde{\psi}^{*}\left(-\frac{1}{2} \nabla^{2}+v_{\mathrm{ne}}\right) \tilde{\psi} d \mathbf{r} \\
& +\iint \frac{|\tilde{\psi}(\mathbf{r})|^{2}|\tilde{\psi}(\mathbf{s})|^{2}}{|\mathbf{r}-\mathbf{s}|} d \mathbf{r} d \mathbf{s},
\end{aligned}
$$

where $v_{\text {ne }}$ is the nucleus-electron Coulomb attraction potential and $\tilde{\psi}$ is a normalized trial orbital. However, by taking into account Eqs. (11) and (7), we can express the RHF energy as a functional depending on both a trial orbital $\tilde{\psi}$ and a trial potential $\tilde{v}$

$$
E_{\mathrm{RHF}}[\tilde{\psi}, \tilde{v}]=2 \int \tilde{\psi}^{*}\left(-\frac{1}{2} \nabla^{2}+v_{\mathrm{ne}}\right) \tilde{\psi} d \mathbf{r}-2 F_{|\tilde{\psi}|^{2}}[\tilde{v}] .
$$

Finally, we obtain the RHF energy via a self-consistentfield (SCF) method by alternating the minimization of this functional with respect to $\tilde{\psi}$ with the maximization with respect to $\tilde{v}$ (note that $F_{|\tilde{\psi}|^{2}}$ enters the functional with a minus sign). The maximization with respect to $\tilde{v}$ is done by the procedure explained above, while the minimization with respect to $\tilde{\psi}$ is performed by solving the Schrödinger equation [17] for the Hamiltonian,

$$
-\frac{1}{2} \nabla^{2}+v_{\mathrm{ne}}+\tilde{v}
$$

with the same map $\mathbf{u}=f(\mathbf{r})$ used for both actions.

We have tested our approach on three two-electron systems: the helium atom and the $\mathrm{H}_{2}$ and $\mathrm{H}_{3}{ }^{+}$molecules. The results are given in Table I, about which we offer some remarks.

(i) As expected, the error decreases steadily as $N$, the number of basis functions per dimension, increases.

(ii) The errors are mostly (but not always) positive. Negative errors appear because we are maximizing with respect to the trial potential $\tilde{v}$, so that an approximate energy below the exact one is perfectly possible. The sign of the error is, however, a secondary matter, because, regardless of sign, the error is still second-order both for the trial orbital $\tilde{\psi}$ and the trial potential $\tilde{v}$.

(iii) Our choices for the map $\mathbf{u}=f(\mathbf{r})$ and for the Coulomb weight $\mu$ are able to yield better than milli-Hartree accuracy with a modest $N$ in all cases. It should be stressed that the same map is good enough for solving both Schrödinger's equation [17] and Poisson's equation.

(iv) Molecules, as well as atoms, are successfully handled by our procedure. There is no need to invoke the supercell 
TABLE I. Errors in the total RHF energy (in a.u.) of some systems estimated as $E_{\mathrm{RHF}}-E_{\mathrm{RHF}}^{\mathrm{ref}}$, where $E_{\mathrm{RHF}}$ is the RHF energy computed by the present method (including nucleus-nucleus repulsion energy), and $E_{\mathrm{RHF}}^{\text {ref }}$ is some accurate reference RHF energy. $N_{u_{1}}, N_{u_{2}}$, and $N_{u_{3}}$ are all set to $N$, so that the total number of basis functions is $N^{3}$. The Coulomb weight parameter has been set to the same value $\lambda_{C}=1$ a.u. in all cases. Technical details for each system are as follows: He: $E_{\mathrm{RHF}}^{\mathrm{ref}}=-2.861679995$ a.u. [34]. Map parameters: $\lambda_{x}=\lambda_{y}=$ $\lambda_{z}=0.9, L_{x}=L_{y}=L_{z}=1$, and $\gamma=0$. Geometry: the nucleus is located at the origin. $\mathbf{H}_{2}: E_{\mathrm{RHF}}^{\mathrm{ref}}=-1.1336295715$ a.u. [35]. Map parameters: $\lambda_{x}=\lambda_{y}=1, \lambda_{z}=1.22066, L_{x}=L_{y}=L_{z}=1$, and $\gamma=0.2$. Geometry: the nuclei are located along the $Z$ axis, one at $z=R / 2$ and the other at $z=-R / 2$, where $R=1.40$ a.u. is the internuclear distance [35]. $\mathbf{H}_{3}^{+}: E_{\mathrm{RHF}}^{\mathrm{ref}}=-1.300400141$ a.u. [36]. Map parameters: $\lambda_{x}=\lambda_{y}=1.20355, \lambda_{z}=1, L_{x}=L_{y}=L_{z}=1$, and $\gamma=0.2$. Geometry: the nuclei are located on the $X Y$ plane forming an equilateral triangle with the center at the origin, one nucleus over the $Y$ axes, and a side length of 1.6405 a.u. [36].

\begin{tabular}{lccc}
\hline \hline$N$ & $\mathrm{He}$ & $\mathrm{H}_{2}$ & $\mathrm{H}_{3}^{+}$ \\
\hline 4 & $+2.2 \times 10^{-1}$ & $+4.2 \times 10^{-1}$ & $+5.1 \times 10^{-1}$ \\
6 & $+7.4 \times 10^{-2}$ & $+1.7 \times 10^{-1}$ & $+1.6 \times 10^{-1}$ \\
8 & $+2.9 \times 10^{-2}$ & $+5.5 \times 10^{-2}$ & $+4.5 \times 10^{-2}$ \\
10 & $+1.7 \times 10^{-2}$ & $+1.8 \times 10^{-2}$ & $+1.7 \times 10^{-2}$ \\
12 & $+9.5 \times 10^{-3}$ & $+5.6 \times 10^{-3}$ & $+9.9 \times 10^{-3}$ \\
14 & $+4.8 \times 10^{-3}$ & $+2.3 \times 10^{-3}$ & $+6.6 \times 10^{-3}$ \\
16 & $+2.3 \times 10^{-3}$ & $+8.7 \times 10^{-4}$ & $+2.5 \times 10^{-3}$ \\
18 & $+1.8 \times 10^{-3}$ & $+3.9 \times 10^{-4}$ & $+7.9 \times 10^{-4}$ \\
20 & $+8.9 \times 10^{-4}$ & $-6.8 \times 10^{-4}$ & $-3.8 \times 10^{-4}$ \\
\hline \hline
\end{tabular}

approximation (or alternatives [31] to it) nor to use pseudopotentials.

\section{CONCLUSIONS}

We have described a procedure for solving Poisson's equation and computing Coulomb energies. Our approach is based on the variational (second-order error) optimization of a functional $F_{\rho}[\xi]$ given in Eq. (9), where the solutions are expressed by the product of a Coulomb weight $\mu$ times a planewave expansion depending on a new coordinate system $\mathbf{u}=$ $f(\mathbf{r})$, as detailed by Eqs. (12) and (13). The main conclusions are as follows:

(i) The method is variational, meaning that the approximated Coulomb energy will have a second-order error with respect to the trial potential error (for nonvariational methods, such as finite-difference methods, the error in the Coulomb energy will be first-order).

(ii) The long-range tail typical of a Coulomb potential is built-in in the solution, thanks to our choice for the Coulomb weight $\mu$ given in Eq. (24).

(iii) Our choice for the map $\mathbf{u}=f(\mathbf{r})$ is able to deal successfully with nonperiodic systems such as atoms or molecules without having to resort to the supercell approach or other alternatives [31].

(iv) The same map $f$ can solve successfully both Poisson's equation and Schrödinger's equation, allowing one to obtain both Coulomb potentials and molecular orbitals, and thus making possible the use of the described method to perform all-electron quantum chemical calculations in atoms and molecules.

(v) As a proof of concept, our implementation is able to achieve, with a moderate number of plane waves, better than milli-Hartree accuracy for the Hartree-Fock energy of twoelectron systems such as the helium atom and the $\mathrm{H}_{2}$ and $\mathrm{H}_{3}{ }^{+}$ molecules.

This method for solving Poisson's equation, together with another variational method [17] for solving Schrödinger equation, would allow variational all-electron quantum chemical calculations for atoms and molecules. The resulting procedure could combine the accuracy of Gaussian basis sets used by quantum chemists with the efficiency and nice scaling properties of plane-wave basis sets used by solid-state physicists.
[1] F. Gygi, Europhys. Lett. 19, 617 (1992).

[2] F. Gygi, Phys. Rev. B 48, 11692 (1993).

[3] F. Gygi, Phys. Rev. B 51, 11190 (1995).

[4] F. Gygi and G. Galli, Phys. Rev. B 52, R2229(R) (1995).

[5] D. R. Hamann, Phys. Rev. B 51, 7337 (1995).

[6] D. R. Hamann, Phys. Rev. B 51, 9508 (1995).

[7] D. R. Hamann, Phys. Rev. B 54, 1568 (1996).

[8] D. R. Hamann, Phys. Rev. B 63, 075107 (2001).

[9] G. Zumbach, N. A. Modine, and E. Kaxiras, Solid State Commun. 99, 57 (1996).

[10] N. A. Modine, G. Zumbach, and E. Kaxiras, Phys. Rev. B 55, 10289 (1997).

[11] A. Devenyi, K. Cho, T. A. Arias, and J. D. Joannopoulos, Phys. Rev. B 49, 13373 (1994).

[12] E. Fattal, R. Baer, and R. Kosloff, Phys. Rev. E 53, 1217 (1996).

[13] G. J. Pearce, T. D. Hedley, and D. M. Bird, Phys. Rev. B 71, 195108 (2005).

[14] J. M. Pérez-Jordá, Phys. Rev. A 52, 2778 (1995).

[15] J. M. Pérez-Jordá, Phys. Rev. B 58, 1230 (1998).
[16] J. M. Pérez-Jordá, J. Chem. Phys. 132, 024110 (2010).

[17] J. M. Pérez-Jordá, J. Chem. Phys. 135, 204104 (2011).

[18] J. I. Rodríguez, D. C. Thompson, P. W. Ayers, and A. M. Köster, J. Chem. Phys. 128, 224103 (2008).

[19] D. Dundas, J. Chem. Phys. 136, 194303 (2012).

[20] S. F. Boys, Proc. R. Soc. London, Ser. A 200, 542 (1950).

[21] M. C. Payne, M. P. Teter, D. C. Allan, T. A. Arias, and J. D. Joannopoulos, Rev. Mod. Phys. 64, 1045 (1992).

[22] A. D. Becke and R. M. Dickson, J. Chem. Phys. 92, 3610 (1990).

[23] T. L. Beck, Rev. Mod. Phys. 72, 1041 (2000).

[24] T. E. Simos and P. S. Williams, Comput. Chem. 23, 513 (1999).

[25] J. E. Pask, B. M. Klein, P. A. Sterne, and C. Y. Fong, Comput. Phys. Commun. 135, 1 (2001).

[26] H.-S. Lee and M. E. Tuckerman, J. Phys. Chem. A 110, 5549 (2006).

[27] R. Alizadegan, K. J. Hsia, and T. J. Martinez, J. Chem. Phys. 132, 034101 (2010). 
[28] R. J. Harrison, G. I. Fann, T. Yanai, Z. Gan, and G. Beylkin, J. Chem. Phys. 121, 11587 (2004).

[29] B. Natarajan, M. E. Casida, L. Genovese, and T. Deutsch, in Theoretical and Computational Developments in Modern Density Functional Theory, edited by A. K. Roy (Nova Science, Hauppauge, New York, 2012), pp. 313-356.

[30] J. W. Cooley and J. W. Tukey, Math. Comput. 19, 297 (1965).

[31] L. Genovese, T. Deutsch, A. Neelov, S. Goedecker, and G. Beylkin, J. Chem. Phys. 125, 074104 (2006).
[32] A. Szabo and N. S. Ostlund, Modern Quantum Chemistry (Dover, New York, 1996).

[33] I. Shavitt, in Methods in Computational Physics, edited by B. Alder, S. Fernbach, and M. Rotenberg (Academic, New York, 1963), Vol. 2, pp. 1-45.

[34] C. F. Bunge, J. A. Barrientos, A. V. Bunge, and J. A. Cogordan, Phys. Rev. A 46, 3691 (1992).

[35] F. Jensen, J. Chem. Phys. 110, 6601 (1999).

[36] F. Jensen, Theor. Chem. Acc. 104, 484 (2000). 